\title{
Transparent Sheath \\ for Neuroendoscopic Intracerebral Hematoma Surgery
}

\author{
Daisuke Suyama, Jun Hiramoto, Kei Yamashiro, \\ Yasuhiro Yamada, Tsukasa Kawase, and Yoko Kato
}

\subsection{Introduction}

Neuroendoscopic procedures for spontaneous cerebral hemorrhage have recently increased. This is a result of the reports published by Nishihara et al. in 2000 [1], describing how to use the neuroendoscopic to evacuate the intracerebral hematoma by using a transparent sheath. Following this, 300 surgical cases of spontaneous intracerebral hemorrhage have been performed with an endoscope in our institution, and in related facilities, between 2000 and 2015. This procedure is commonly performed with only one or two burr holes under local anesthesia. If the

\footnotetext{
D. Suyama $(\bowtie)$

Department of Neurosurgery, Fuchu Keijinkai Hospital, Tokyo, Japan

Department of Neurosurgery, Fujita Health University, Banbuntane Hotokukai Hospital, Nagoya city, Aichi, Japan

e-mail: daisuke@suyama.org

\section{J. Hiramoto}

Department of Neurosurgery, Fuchu Keijinkai Hospital, Tokyo, Japan

K. Yamashiro · Y. Yamada · T. Kawase Department of Neurosurgery, Fujita Health University, Banbuntane Hotokukai Hospital, Nagoya city, Aichi, Japan

e-mail: kawasemi@hm8.aitai.ne.jp

Y. Kato

Department of Neurosurgery, Fujita Health University, Toyoake, Aichi, Japan

e-mail: kyoko@fujita-hu.ac.jp
}

transparent sheaths are introduced into hematoma safely, endoscopic procedures will be successful in any location of spontaneous hematomas.

\subsection{Preoperative Preparation}

Prior to the surgery, if the hematoma is at unusual location, the patient needs an angiography or at least three-dimensional computerized tomographic angiography (3D-CTA) or magnetic resonance angiography (MRA) to rule out the possibility of vascular malformation, such as arteriovenous malformation (AVM), aneurysm, or dural AV fistula.

To decide on surgical approach, 3D-CT was performed with scalp marker. For deep-seated hematoma like thalamic hemorrhage, we used stereotaxic system or navigation system in order to insert transparent sheath.

\subsubsection{Surgical Tools}

For this surgery, we use endoscope, suction tube, and transparent sheath. There are several different types of transparent sheath depending on the case. Some cases require small diameter of $5 \mathrm{~mm}$, but some require $10 \mathrm{~mm}$. Soft blood clot may need small suction of $2 \mathrm{~mm}$, but hard clot may need bigger one such as $4 \mathrm{~mm}$. Rigid and flexible 

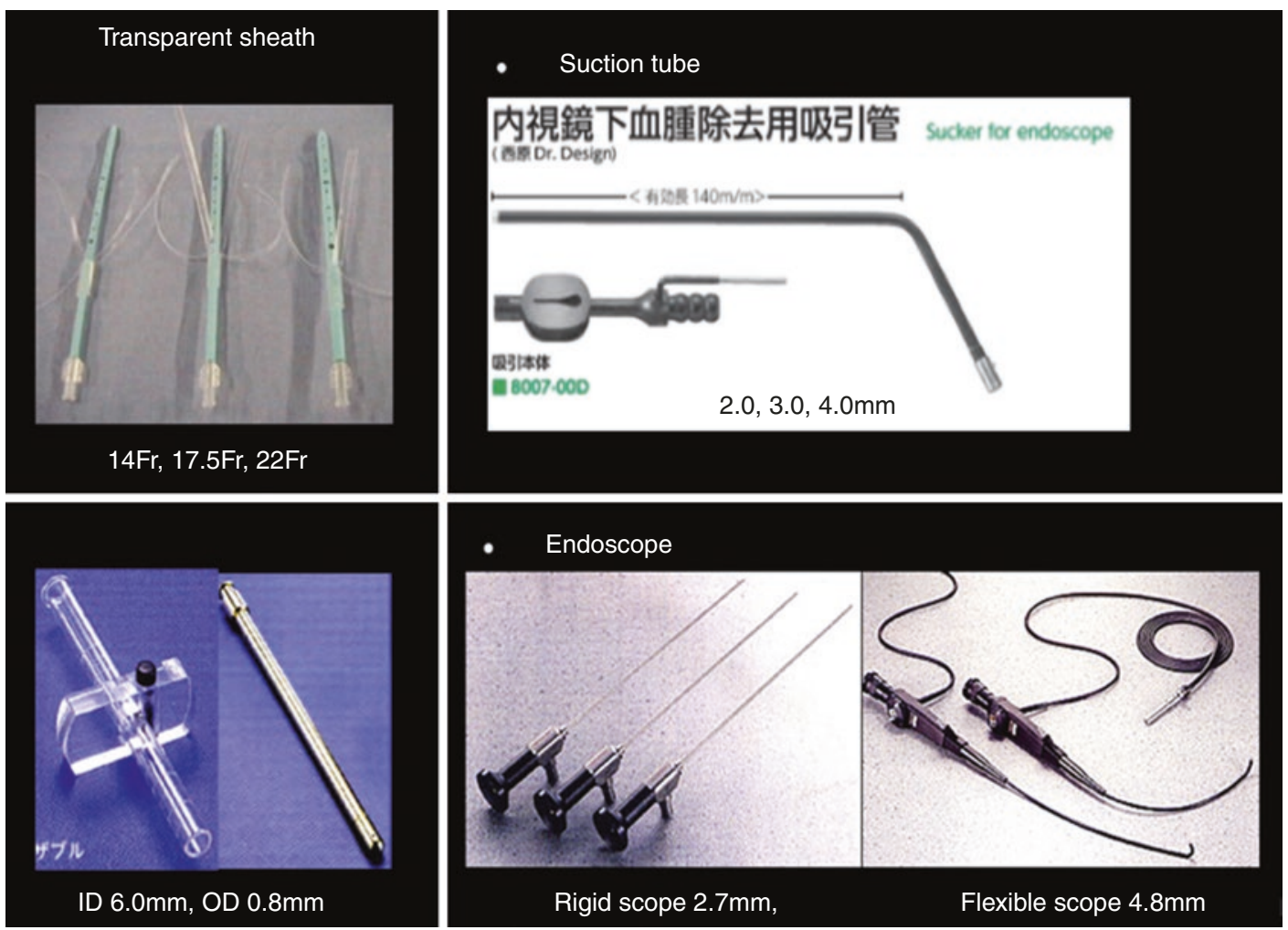

Fig. 23.1 Surgical tools for endoscopic evacuation of intracerebral hematoma

endoscopes are available and their diameter is 2.7 and $4.8 \mathrm{~mm}$ (Fig. 23.1).

\subsection{Steps of the Surgery}

\subsubsection{Burr Hole and Hematoma Evacuation}

Prior to incision, we need to infiltrate the skin with local anesthesia, and it is very important to infiltrate the periosteum. The periosteum is very sensitive to pain. Linier incision is made and secured with self-retaining retractor. A burr hole is made and should be large enough for the transparent sheath; the sheath is inserted using CT-stereotaxic or navigation guidance until it gets into the hematoma. The transparent sheath (Fig. 23.1) with allows our vison along the tunnel, with the scope and suction tube in it, we will be able to suck out the blood clot. The tube is moveable and has a slippery surface to prevent injury to the brain. If we find the source of bleeding vessel, it could be coagulated with touching the monopolar to the suction tube on the source of bleeding (Fig. 23.2).

\subsubsection{Irrigation}

When the hematoma is evacuated, we may irrigate the hematoma bed with warm normal saline or ringer solution. Sometimes, when we expand the cavity with the fluid, some residual hematoma is also washed out. The warm fluid usually helps with the local hemostasis, and it's very helpful especially with small oozing blood. Condition with significant bleeding requires bipolar or monopolar. After things are done, the transparent sheath is removed gently and the tract also needs to be washed with warm fluid. No drain is necessary in the hematoma cavity (Fig. 23.3). 

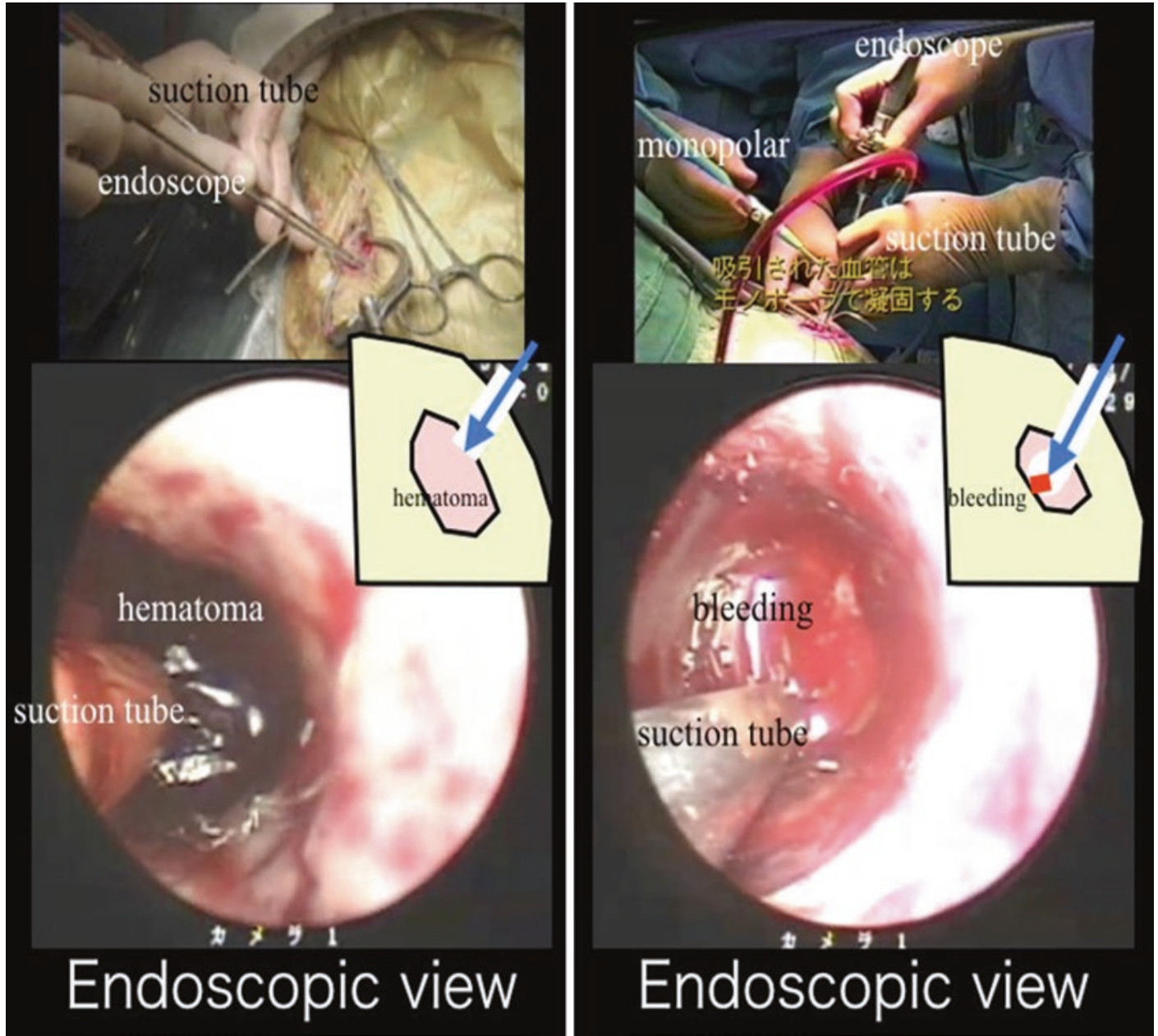

Fig. 23.2 Hematoma evacuation: left endoscopic evacuation of the hematoma, right endoscopic found and control the source of hemorrhage

\subsection{Specific Surgical Technique}

The use of endoscope to evacuate the intracerebral hematoma began with endoscopic-assisted stereotaxic aspiration of hematoma, which is popular before $2000[2,3]$. In contrast to that, recently, the use of endoscope with transparent sheath for evacuation of hematoma is the major role because it provides the direct vision compared to the stereotaxic aspiration surgery. The transparent sheath is movable and provides a clear orientation around the tube. This technique has gain popularity very fast, and it could be done with small incision and with small craniotomy (or burr hole). The entry point for different locations is very important, such as putaminal hematoma, thalamic hematoma, lobar hematoma, and cerebellar hematoma.

\subsubsection{Putaminal Hemorrhage}

In putaminal hematoma, the burr-hole position should be put at the upper end of the hematoma to the closest vertical trajectory from the cranium. Removing the hematoma and being able to see the brain margin will help the orientation. The hard clot should not be pulled and better to be left 

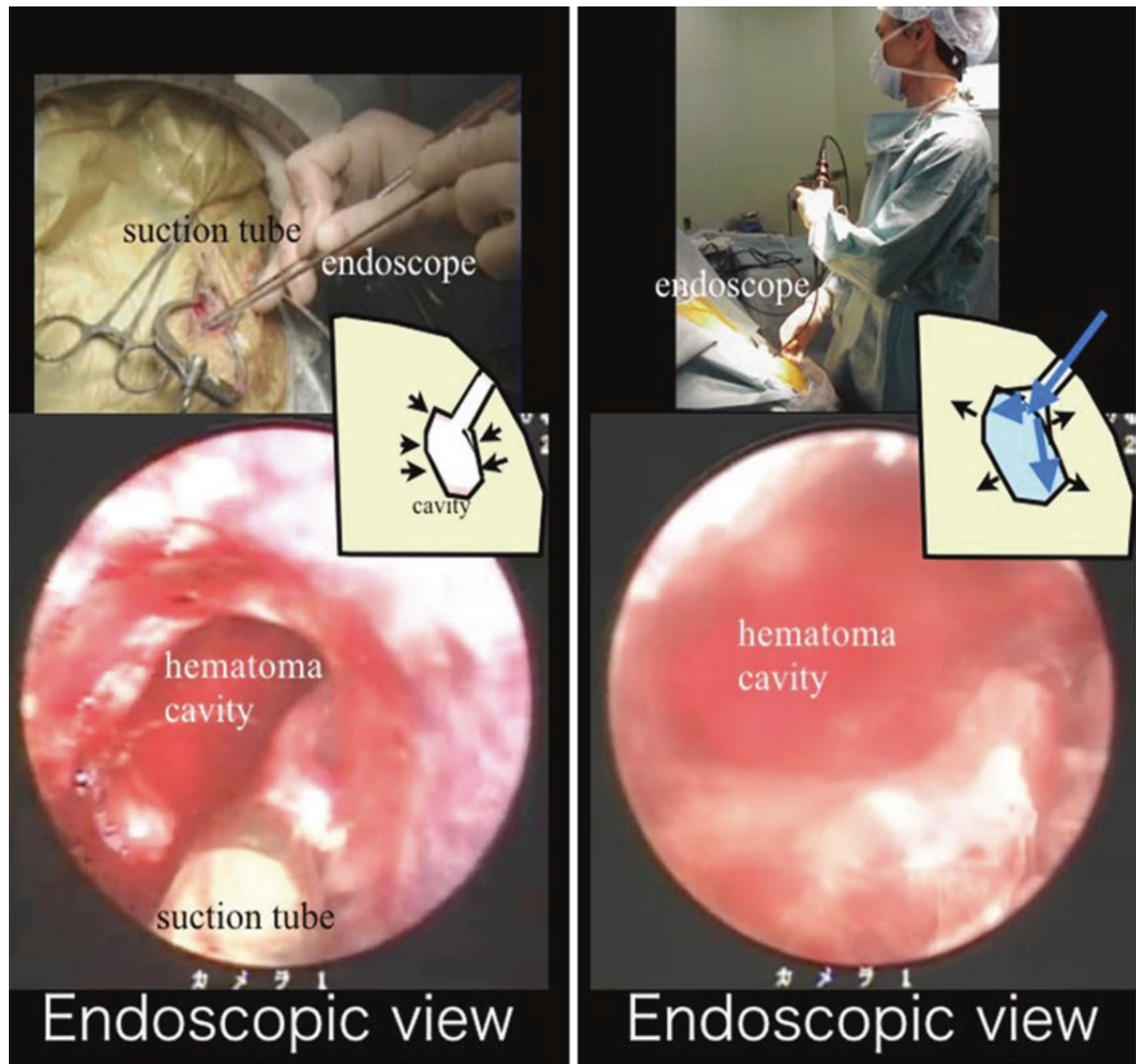

Fig. 23.3 Irrigation of hematoma cavity: left rigid scope view, right flexible scope view

there. After the hematoma removal, we may irrigate the cavity with warm saline (Fig. 23.4).

\subsubsection{Thalamic Hemorrhage}

In thalamic hematoma, we have to consider the blood both in the ventricle and in the parenchyma. Also, we need to remember and imagine the location of internal capsule to the blood clot. The surgical approach could be from anterior or posterior transventricular and try to avoid injury to internal capsule, although it's probably already injured by the hemorrhage. But we need to try our best to avoid making more injury to the internal capsule, if there are still some. After evacuating the thalamic hematoma, the blood clot in the ventricle could be aspirated gently. Sometimes we need a flexible scope (Fig. 23.5). But even with rigid scope and lots of irrigation, we may clear the hematoma mostly.

\subsubsection{Lobar Hemorrhage}

In lobar hemorrhage, the trajectory for lobar hematoma should be planned with navigation or for 3D-CT with scalp marker (Fig. 23.6). 
Fig. 23.4 CT scan of putaminal hemorrhage: $O$ point of entry, $X$ longitudinal axis of the hematoma

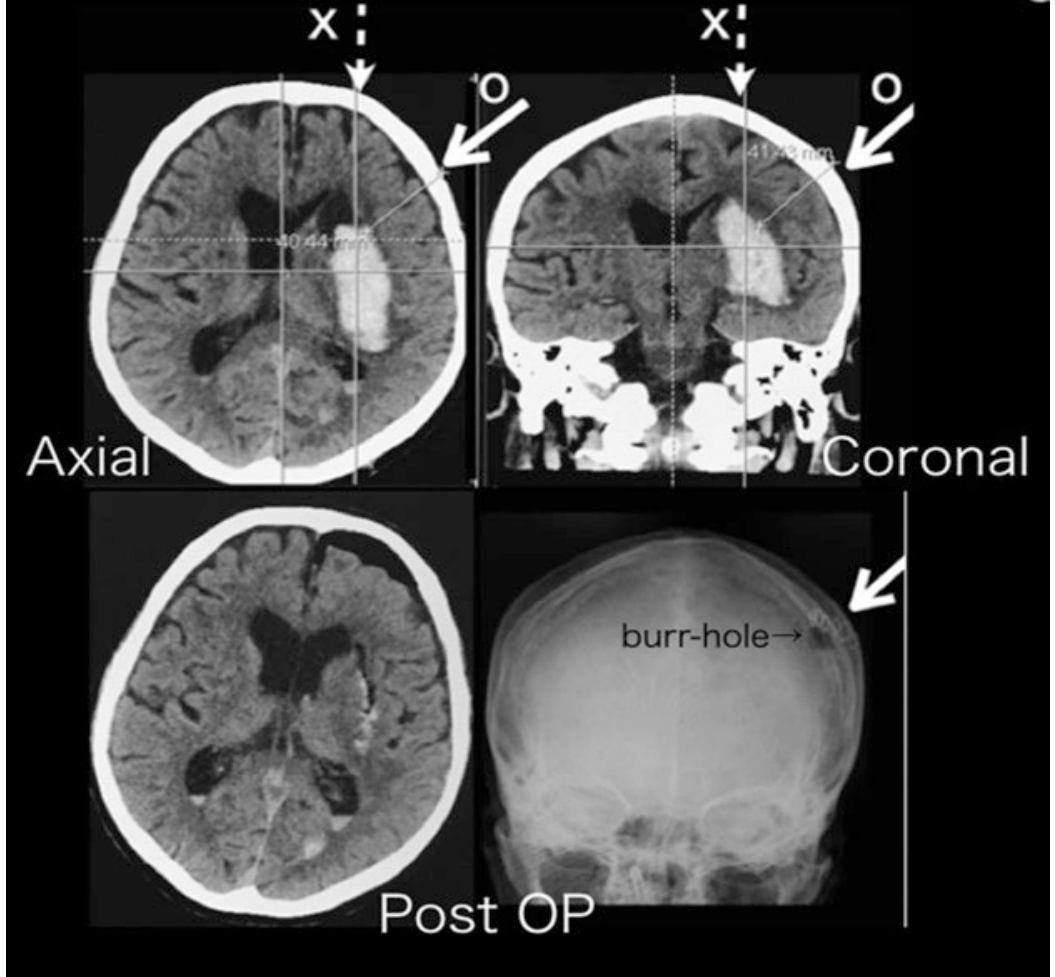

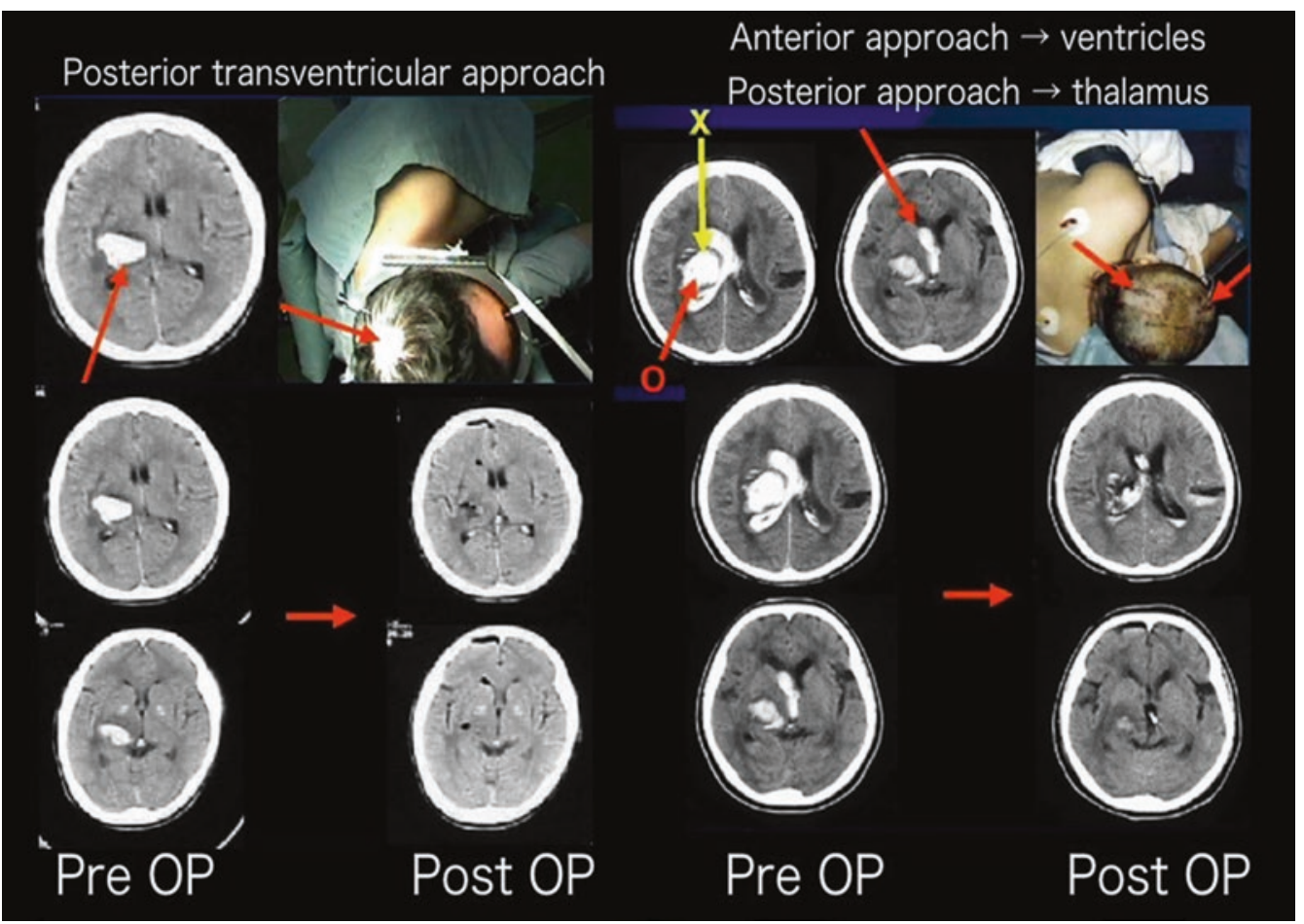

Fig. 23.5 Thalamic hemorrhage: left, posterior transventricular removal of the hematoma; right, coming from posterior with rigid scope to remove the thalamus hema- toma and coming from anterior horn with flexible scope to remove the intraventricular hematoma 


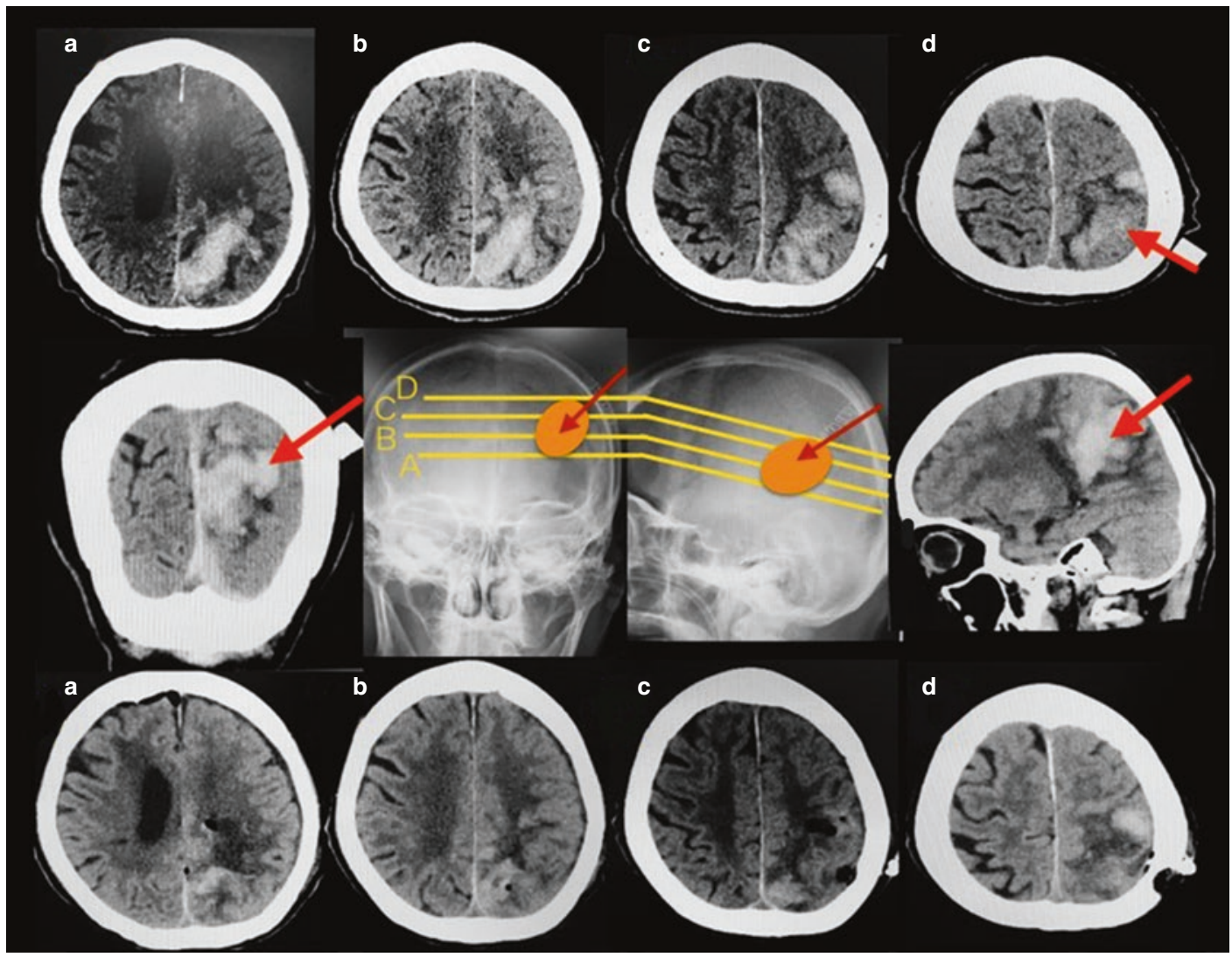

Fig. 23.6 CT scan of lobar hemorrhage, before surgery and after surgery (lower part)

\subsubsection{Cerebellar Hemorrhage}

In cerebellar hemorrhage, burr hole position is very important but it is difficult to decide a proper position only by preoperative scalp surface marker; a midpoint of the line between the inion and the mastoid tip. Burr-hole position should be measured from the foramen magnum on the skull surface during surgery. It is the most reliable marker during surgery, to make a burr hole on the inferior nuchal line in the distance of $3 \mathrm{~cm}$ from the foramen magnum (Fig. 23.7).

\subsection{Expert Opinion/Suggestion to Avoid Complication}

In our cases of putaminal hemorrhage, two cases we switched craniotomy. One case is due to enlargement of the hematoma after surgery, and this probably due to either insufficient hemostasis or rebleeding after surgery. The second case was due to a small AVM that we misdiagnosed at the beginning. It was described by Yasagil in 1987 [4]. The diagnose was proved during craniotomy and the pathology confirmed it. We highly recommend to screen every possible case, especially if the location and the clinical presentation are not typical for hypertensive hemorrhage. If there is a massive bleeding during hematoma evacuation, do not hesitate to switch to craniotomy for hematoma removal. All of such thing should be mentioned in the consent prior to surgery, including the provable micro-AVM [5].

In our cases of thalamic hemorrhage, we have two cases with intraventricular venous; both cases were using an anterior transventricular approach for thalamic hematoma. Therefore, our opinion is considering that a posterior approach is probably better than an anterior approach to 

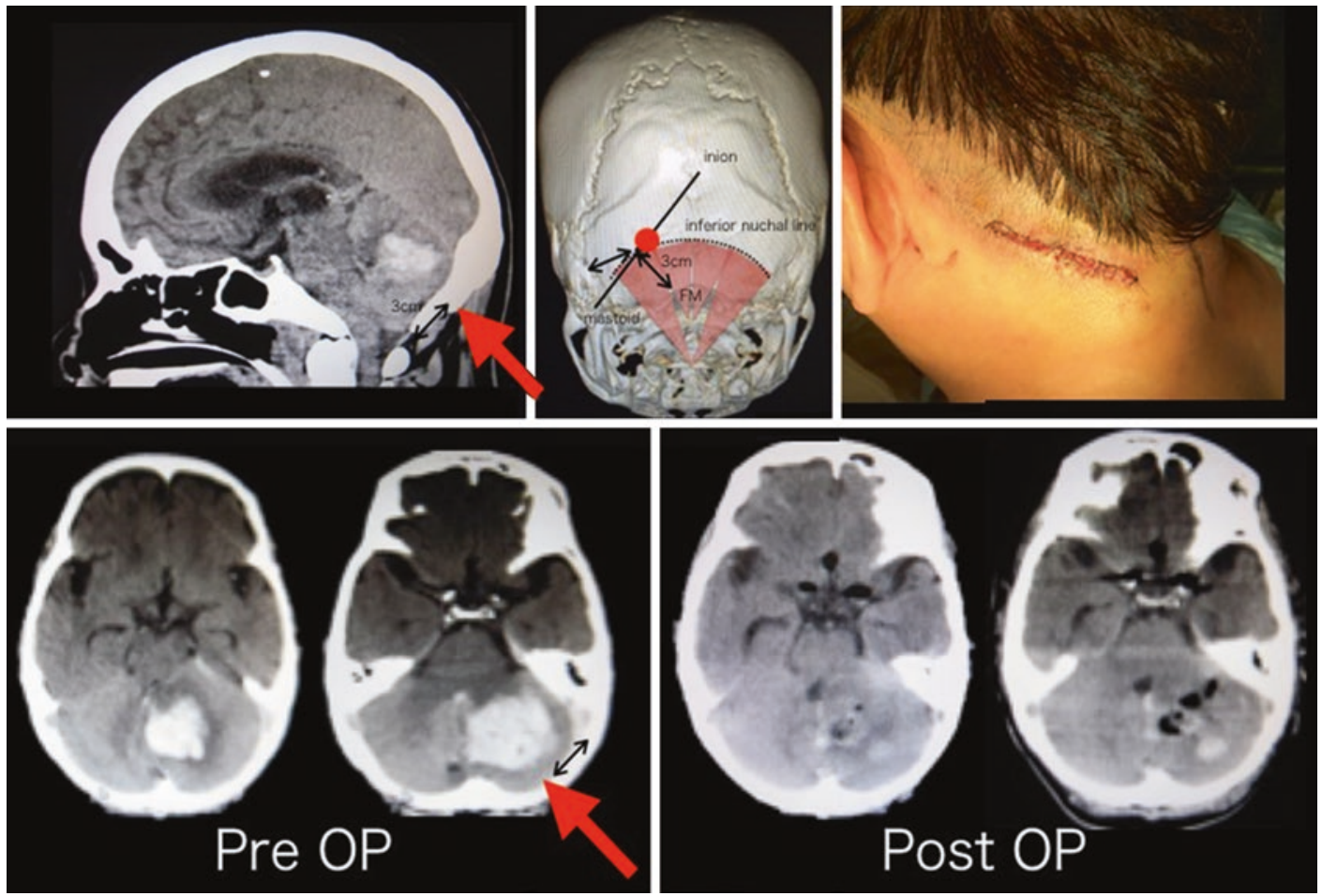

Fig. 23.7 Cerebellar hemorrhage, before and after surgery

remove the thalamic hematoma to avoid injury of intraventricular veins.

Transparent sheath for endoscopic evacuation of intracerebral hematoma will become more and more popular. Once the surgeon becomes familiar and comfortable with this technique, they will less likely go back to the conventional approach with bigger incision and bigger wound. The important points of this surgery is the placement of the burr hole so the track will provide the best direction for hematoma evacuation.

\section{References}

1. Nishihara T, Teraoka A, Morita A, et al. A transparent sheath for endoscopic surgery and its application in surgical evacuation of spontaneous intracerebral hematomas. J Neurosurg. 2000;92:1053-5.

2. Auer LM, Holzer P, Ascher PW, et al. Endoscopic neurosurgery. Acta Neurochir. 1988;90:1-14.

3. Kuroda K, Ogawa A. Comparative studies of surgical treatments for intracerebral hemorrhage: mainly on an echo-guide aspiration and a CT-guide aspiration with neuroendoscopy. Jpn J Neurosurg. 1999;8:100-5.

4. Yasargil MG. Microneurosurgery, vol 3B. Stuttgart: Thieme; 1987. p. 18-9.

5. Suyama D, Ito K, Tanii M, et al. A difficult case of endoscopic neurosurgery for intracerebral hemorrhage due to micro-arteriovenous malformation. Surg Cereb Stroke (Jpn). 2003;31:361-4. 
Open Access This chapter is licensed under the terms of the Creative Commons Attribution 4.0 International License (http://creativecommons.org/licenses/by/4.0/), which permits use, sharing, adaptation, distribution and reproduction in any medium or format, as long as you give appropriate credit to the original author(s) and the source, provide a link to the Creative Commons license and indicate if changes were made.

The images or other third party material in this chapter are included in the chapter's Creative Commons license, unless indicated otherwise in a credit line to the material. If material is not included in the chapter's Creative Commons license and your intended use is not permitted by statutory regulation or exceeds the permitted use, you will need to obtain permission directly from the copyright holder.

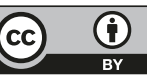

